\title{
ÉQUATIONS DE NAVIER STOKES RÉDUITES POUR LES ÉCOULEMENTS BIOMÉCANIQUES: ÉCHELLES CARACTÉRISTIQUES ET CONDITIONS AUX LIMITES.
}

\author{
P.-Y. LAGRÉE ${ }^{1}$
}

\begin{abstract}
A simplified system issued from Navier Stokes equations is presented. Its link with other asymptotical methods is explained. It is solved for applications in stenosed flows in arteries and in the glottis.

Résumé. Un système d'équations simplifiées est présenté, son lien avec d'autres méthodes asymptotiques est discuté. Ce système est résolu numériquement sur des exemples dans le cas des artères sténosées et de la glotte.
\end{abstract}

\section{INTRODUCTION}

Un des problèmes rencontrés lors de la résolution des équations de Navier Stokes dans des artères sténosées est celui des conditions aux limites. En résolvant les équations de Navier Stokes de manière simplifiée avec des méthodes issues du cadre de la couche limite (donc à grand nombre de Reynolds), nous allons montrer que le profil de vitesse exact en amont est de peu d'importance (seul compte le flux total) et que de plus la sortie est un résultat du calcul (qu'il s'agisse du profil de vitesse ou de la valeur finale de la pression).

Pour illustrer ceci nous considérons ici l'écoulement dans un canal axisymétrique présentant une constriction avec en vue des applications dans des artères sténosées, le même type d'équations peut être écrit en 2D plan pour des applications dans la glotte.

Nous revisitons en fait les travaux classiques de Smith en montrant que les équations RNS/Prandtl contiennent la plupart des effets présents dans les équations de double et triple couche.

\section{1. Équations RNS/Prandtl (Ou RNSP(x))}

\subsection{Le système en axisymétrique}

Partant des équations de Navier Stokes (NS) en régime stationnaire laminaire incompressible et axisymétrique dans un tuyau de rayon initial $R_{0}$, on suppose que les variations transverses de la vitesse longitudinale (d'échelle construite avec la vitesse $U_{0}$, tel que le flux soit $\left.Q=\pi R_{0}^{2} U_{0}\right)$ sont justement d'échelle $R_{0}$. On choisit ensuite

\footnotetext{
1 Lab. de Modélisation en Mécanique, UMR CNRS 7607, B 162,

Université Paris 6, 75252 Paris FRANCE

Tel (33)144272559 - Fax (33)144275259 - pyl@ccr.jussieu.fr
} 
l'échelle longitudinale $\left(R_{0} R e\right.$ bien plus grande que $R_{0}$ où $R e=U_{0} R_{0} / \nu>>1$ est le nombre de Reynolds) de manière à garder le maximum de termes dans les équations. Ainsi, avec:

$$
x=\bar{x} R_{0} R e, r=\bar{r} R_{0}, u=U_{0} \bar{r}, v=\frac{U_{0}}{R e} \bar{v} \quad \text { et } \quad p=\rho_{0} U_{0}^{2} \bar{p}
$$

(la référence de pression est prise en $\bar{x}=0$ ), le système suivant est obtenu lorsque $R e \rightarrow \infty$ :

$$
\begin{gathered}
\frac{\partial}{\partial \bar{x}} \bar{u}+\frac{\partial}{\bar{r} \partial \bar{r}} \bar{r} \bar{v}=0 \\
\left(\bar{u} \frac{\partial}{\partial \bar{x}} \bar{u}+\bar{v} \frac{\partial}{\partial \bar{r}} \bar{u}\right)=-\frac{\partial \bar{p}}{\partial \bar{x}}+\frac{\partial}{\bar{r} \partial \bar{r}}\left(\bar{r} \frac{\partial}{\partial \bar{r}} \bar{u}\right), \quad 0=-\frac{\partial \bar{p}}{\partial \bar{r}} .
\end{gathered}
$$

Il s'agit d'équations cohérentes asymptotiquement que l'on peut appeler $\operatorname{RNSP}(\mathrm{x})$ par référence à "Reduced NS" (de Rubin Himansu [10] qui gardent une variation transverse qui n'a pas de sens asymptotique); mais, il s'agit en fait des équations de Prandtl, d'où la dénomination RNS/Prandtl. On remarque que la pression ne dépend que de $\bar{x}$, elle est constante dans chaque section.

Les conditions aux limites sont:

- condition de symétrie au centre $\left(\partial_{\bar{r}} \bar{u}=0\right.$ et $\bar{v}=0$ en $\left.\bar{r}=0\right)$,

- adhérence $(\bar{u}=\bar{v}=0$ en $\bar{r}=1-\bar{f}(\bar{x})), \bar{f}(\bar{x})$ est la forme de la perturbation de la paroi, dont les échelles sont supposées compatibles),

- un profil d'entrée donné $(\bar{u}(0, \bar{r})$ et $\bar{v}(0, \bar{r}))$, on peut imposer un profil plat $\bar{u}(0, \bar{r})=1, \bar{v}(0, \bar{r})=0$ (ou tout autre profil, dont Poiseuille).

La présence des termes $\bar{u} \frac{\partial}{\partial \bar{x}} \bar{u}$ et $\frac{\partial}{\bar{r} \partial \bar{r}}\left(\bar{r} \frac{\partial}{\partial \bar{r}} \bar{u}\right)$ suggère que ces équations peuvent se résoudre en marchant en $\bar{x}$ et qu'ainsi il n'y a pas de condition de sortie, le système semble parabolique.

Les limites d'application de cette remarque sont discutés dans Lagrée \& Lorthois [7] nous rappelons les arguments dans la section suivante.

\subsection{Cas 2D, instationarité}

Le cas 2D est "identique" aux notations près. Le cas instationnaire fait alors intervenir une échelle de temps qui est $R_{0}^{2} / \nu$. Les équations sont présentées aussi dans Lagrée [4] où la paroi bouge.

\section{DÉGÉNÉRESCENCES DE CES ÉQUATIONS}

\section{1. équations IBL}

Partant d'un profil plat en entrée, on voit alors qu'il existe une gamme d'échelles longitudinales pour lesquelles, partant des équations de Navier Stokes ou des équations RNS/Prandtl on obtient alors les équations de couche limite interactives (IBL). Ces équations sont paraboliques en $x$.

\section{2. Équations de double couche stationnaires}

Partant d'un profil de Poiseuille, on peut faire varier les dimensions de la bosse, Les équations de "double couche" s'obtiennent en examinant les équations NS près de la paroi au voisinage de la position de la bosse en utilisant une nouvelle échelle longitudinale. On constate alors (Smith [13], Saintlos \& Mauss [11]) que ces équations sont valides pour des longueurs de bosses plus petites que $R_{0} R e$ et d'épaisseur plus petite que $R_{0}$ jusqu'à une longueur de bosse égale au rayon $R_{0}$ et d'épaisseur $R_{0} R e^{-1 / 3}$. Les équations $\operatorname{RNSP}(\mathrm{x}) \operatorname{permettent}$ de retrouver ces résultats au premier ordre. Il faut noter qu'il existe un cas particulier pour la longueur $R_{0} R e^{1 / 7}$ et la hauteur $R_{0} R e^{-2 / 7}$, tel qu'il peut y avoir une remontée de l'information. En fait dans le cas axisymétrique, cette remontée de l'information disparaît et les équations sont toujours paraboliques en $x$. 


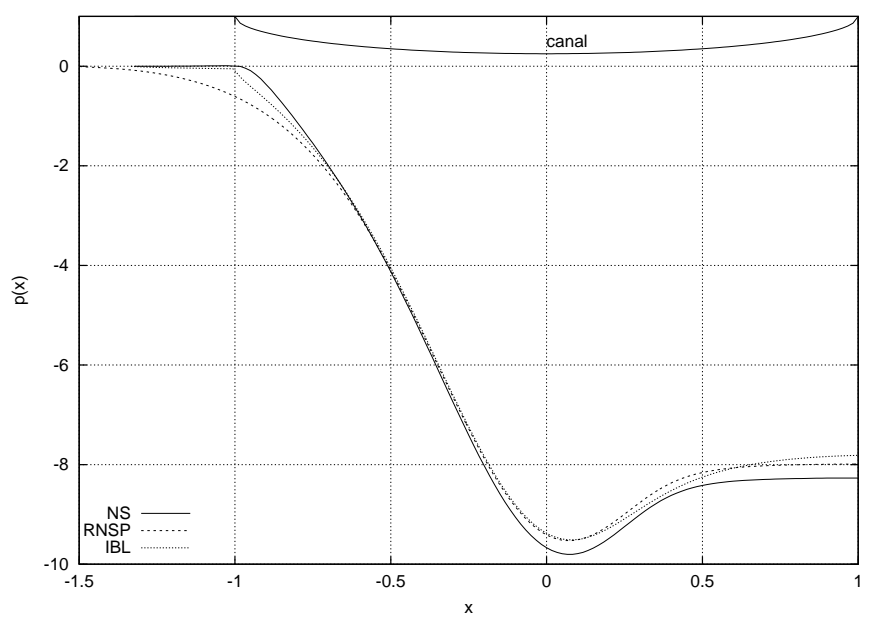

Figure 1. Comparaison entre la pression calculée par Navier Stokes (Castem 2000), IBL (couche limite interactive) et RNSP, en 2D le long d'une sténose symétrique constituée de deux demi-cercles. Pour calculer RNSP on a ajouté un pied doux (pour éviter d'attaquer la sténose qui présente une pente infinie) pour IBL cela n'a pas été la peine. Le degré de sténose est $\alpha=0,75$ et $R e=500$.

\section{3. Équations de double couche instationnaires}

Reprenant les équations de double couche instationnaires de Duck [2], on peut montrer qu'elles sont comprises dans les équations RNSP(x) instationnaires (Lagrée \& Goorman [5]).

\section{4. Équations de triple couche}

Les équations RNSP(x) permettent aussi de retrouver le problème de "triple couche" dans un tuyau lorsqu'il existe un coeur potentiel. Si la position de la bosse $x_{0}$ est telle que $R_{0} R e^{2 / 5}<<x_{0}<<R_{0} R e$, on obtient un problème encore parabolique en x (Ruban \& Timoshin [9]). Si $x_{0}=R_{0} R e^{2 / 5}$ on a alors le problème de triple couche avec comme pont supérieur (upper Deck) toute la largeur du tuyau (Smith [13]), dans ce cas la description $\operatorname{RNSP}(\mathrm{x})$ tombe en défaut.

\section{Applications}

\subsection{Influence aval/amont:}

Les équations RNSP(x) permettent de calculer la longueur d'établissement du régime de Poiseuille (Schlichting [12], Lagrée \& Lorthois [7]), que l'on a comparé à des calculs NS directs (de Bruin et al. [1]). On a aussi vérifié en $2 \mathrm{D}$ plan que si l'on faisait varier la position d'une constriction son influence est très faible en amont.

\subsection{Comparaison à des méthodes intégrales directes}

Le cas des sténoses (les anévrismes peuvent être abordés de la même manière Lagrée [3]) est remarquable. Dans Lagrée \& Lorthois [7] (et Lorthois et al. [8]) il est montré que l'on peut estimer le pic de frottement pariétal par une formule approchée qui permet de retrouver les résultats de calculs NS de la littérature. Le cas de la paroi élastique est examiné dans Lagrée [4] où une comparaison est faite entre une méthode instationnaire et une méthode intégrale. Sur la figure on a comparé la résolution avec une méthode intégrale, les équations RNSP et une solution numérique directe à l'aide du code CASTEM 2000. 


\section{Conclusion}

Dans le cas des tuyaux, le système RNS/Prandtl inclut les jeux d'équations de double couche/ de triple couche/ de couche limite interactive. Le caractère de marche en avant (parabolique en $x$ ) des équations est donc justifié par l'analyse asymptotique. Le gain de temps en calcul est très important, l'adimensionalisation permet en outre d'exhiber les bonnes échelles des phénomènes que ce soit dans une artère sténosée ou dans la glotte.

\section{REFERENCES}

[1] de Bruin B., Lagrée P.-Y., Lorthois S., Vilain C., Veldman A.E.P. (2001): "Comparison of Navier Stokes and Reduced Navier Stokes unsteady computation in a stenosis", Archives of Physiology and Biochemistry Vol 109 sept 2001, p 79.

[2] Duck P.W. (1979): "Viscous flow through unsteady symmetric channels", JFM vol 95, p 4, pp 635-653.

[3] P.-Y. Lagrée (1998): "Écoulement dans un anévrisme: comparaison de différentes méthodes de type couche limite", Archives of Physiology and Biochemistry Vol 106 supp B, septemb 98, p 42.

[4] P.-Y. Lagrée (2000): "An inverse technique to deduce the elasticity of a large artery ", European Physical Journal, Applied Physics 9, pp. 153-163

[5] Lagrée \& Goorman: soumis

[6] P.-Y. Lagrée \& S. Lorthois (2000): "Reduced Navier Stokes in axisymetrical stenoses". 4th EUROMECH Fluid Mechanics Conference (EFMC2000), Eindhoven 19-23 November 2000.

[7] P.-Y. Lagrée \& S. Lorthois: The RNS/Prandtl equations, their link with other asymptotic descriptions, application to the computation of the maximum value of the Wall Shear Stress, submitted.

[8] S. Lorthois, P.-Y. Lagrée, J.-P. Marc-Vergnes \& F. Cassot. (2000): "Maximal wall shear stress in arterial stenoses: Application to the internal carotid arteries", Journal of Biomechanical Egineering, Volume 122, Issue 6, pp. 661-666.

[9] Ruban A.I. \& Timoshin S.N. (1986): "Propagation of perturbations in the boundary layer on the walls of a flat channel", MZG 2, pp. 74-79.

[10] Rubin S.G. \& Himansu A. (1989): "Convergence properties of high Reynolds number separated flow calculations “, Int J. Num meth fluids vol 9 1395-1411.

[11] S. Saintlos \& J. Mauss (1996): "asymptotic modelling for separating boundary layers in a channel", Int. J. Engng. Sci.,Vol 34, No 2, pp201-211.

[12] H. Schlichting (1987): "Boundary layer theory" 7th ed Mc Graw Hill.

[13] F. T. Smith (1976): "Flow through constricted or dilated pipes and channels", part 1 and 2, Q. J. MEch. Appl. Math. vol 29, pp 343- $364 \&$ 365- 376. 